\title{
ANÁLISE TÉCNICA E DE CUSTOS DO TRANSPORTE DE MADEIRA COM DIFERENTES COMPOSIÇÕES VEÍCULARES ${ }^{1}$
}

\author{
Rafael Tonetto Alves², Nilton Cesar Fiedler ${ }^{3}$, Elizabeth Neire da Silva ${ }^{3}$, Eduardo da Silva Lopes ${ }^{4}$ e \\ Flávio Cipriano de Assis do Carmo ${ }^{5}$
}

\begin{abstract}
RESUMO - O transporte de madeira no Brasil é realizado predominantemente pelo modal rodoviário, chegando o frete a representar até $60 \%$ do custo logístico total. Objetivou-se neste trabalho analisar os fatores técnicos e de custos do transporte de madeira com diferentes composições veiculares de carga, na região do Vale do Rio Doce, MG. Realizou-se um estudo de tempos e movimentos do ciclo operacional de transporte realizado nos períodos diurno e noturno, da operação de carregamento e descarregamentode madeira. As análises foram divididas em três regiões de produção de madeira com diferentes distâncias de transporte. Os resultados indicam quea região de Nova Era apresentou o maior tempo produtivo (78,71\%) e o pior improdutivo (9,26\%). Em relação aos custos operacionais, as regionais, que utilizavam o bitrem como veiculo de transporte de madeira. Os valores por $\mathrm{km}$ de transporte encontrados foram $0,341 \mathrm{R} \$ / \mathrm{m}^{3} \cdot \mathrm{km}^{-1}$ para Nova Era e $0,249 \cdot \mathrm{R} \$ / \mathrm{m}^{3} \cdot \mathrm{km}^{-1} \cdot$ para Rio Doce. Para a região de Guanhães, obteve-se o maior custo final da madeira $\left(27,14 \mathrm{R} \$ / \mathrm{m}^{3} \cdot \mathrm{km}^{-1}\right)$.A utilização do tritrem como veículo para transporte de madeira, apresentou os menores custos de $\mathrm{m}^{3}$ transportado por $\mathrm{km}$.
\end{abstract}

Palavras-chave: Transporte de madeira; Custos de transporte; Veículos de transporte.

\section{TECHNICAL ANALYSIS AND TRANSPORTATIONCOSTS OF WOOD WITH DIFFERENT TYPES OF VEHICLES}

\begin{abstract}
Wood transportation in Brazil is predominantly carried by road, and the freight represent up to $60 \%$ of the total logistics cost. The objective of this studywas to analyze the technical factors and costs of transporting woodwith different compositions of vehicular load, in Vale do Rio Doce region, Minas Gerais State. Initially, a time and motion study aboutthe operating cycle transport were conducted, performed atday and nighttime periods, and duringwood loading and unloadingoperations. The analyses were divided into three regions of wood production with different transport distances. The results showed that Nova Era region had the highest percentage of productive time (78.71\%) and the worst unproductive (9.26\%). Regarding operating costs, theregionals used bitrem truck as the vehicleto transportwood. The transport values were $0.341 \mathrm{R} \$ / \mathrm{m}^{3} . \mathrm{km}^{-1}$ for Nova Era, and $0.249 \mathrm{R} \$ / \mathrm{m}^{3} . \mathrm{km}^{-1}$ forRio Doce. The highest final cost of the wood (27.14 R\$/ $\left.\mathrm{m}^{3} . \mathrm{km}^{-1}\right)$ was obtained in the ofregionGuanhães. The use oftritrem as a vehicle totransportwood had the lowest costs per $\mathrm{m}^{3}$ transported per $\mathrm{km}$.
\end{abstract}

Keywords: Wood transport; Transport costs; Transport vehicles.

\footnotetext{
${ }^{1}$ Recebido em 04.09.2012 aceito para publicação em 27.08.2013.

${ }^{2}$ Eldorado Brasil Celulose S.A., Eldorado, Brasil. E-mail: <rafafloresteiro@hotmail.com>.

${ }^{3}$ Departamento de Ciências Florestais e da Madeira da Universidade Federal do Espírito santo, ES, Brasil. E-mail: <fiedler@pq.cnpq.br> e<elizabeth@cca.ufes.br>.

${ }^{4}$ Universidade Estadual do Centro-Oeste, Centro de Ciências Agrárias e Ambientais, Departamento de Engenharia Florestal. E-mail: <eslopes@pq.cnpq.br>.

${ }^{5}$ Programa de Pós-Graduação em Mestrado em Ciências Florestais pelo Programa de Ciências Florestais da UFES, ES, Brasil.<flaviocipriano@hotmail.com>.
} 


\section{INTRODUÇÃO}

Berger et al. (2003) e Rodrigues (2007) afirmam que o transporte é um dos fatores de composição do custo logístico de maior valor, e nas nações desenvolvidas, somente os fretes costumam consumir aproximadamente $60 \%$ do custo logístico total, estando entre $9 \%$ a $10 \%$ do produto nacional bruto, justificando a busca pela eficiência e qualidade em todas as fases do processo.

No Brasil, o transporte rodoviário corresponde a 59\% da carga transportada, enquanto o transporte ferroviário detém 24\%. Segundo Machado et al. (2009), em outros países com dimensões similares ao Brasil o transporte ferroviário tem maior força como na Rússia (81\%) e Canadá (46\%).

No Brasil, a grande maioria do transporte florestal é realizada pelo modal rodoviário, devido ao histórico e tendência nacional. Além disso, alguns fatores contribuem para esta situação: extensa malha viária, oferta de diferentes tipos de veículos, baixo valor de instalação quando comparado a outros modais existentes (MACHADO et al., 2009).Para um melhor planejamento do tipo de veiculo a ser utilizado no transporte de madeira, deve ser levado em consideração a distância do local de carregamento (margens dos talhões ou pátios intermediários) até a indústria, a quantidade de madeira a ser transportada e a capacidade de carga de cada veículo; Estes fatores influenciarão no custo final da madeira posto em fábrica.

Essa facilidade de escolha de veículos com diferentes capacidades de cargas e rotas permitidas pelo modal rodoviário passa a tornar-se um problema, quando não se faz a escolha do veículo e planejamento adequado da operação. No caso específico de veículos de transporte florestal, um dos fatores que mais contribuem para o aumento dos custos operacionais é o desgaste prematuro das estradas florestais, por acarretar maior consumo de pneus e peças, e contribuir de forma negativa à velocidade de deslocamento (BURLA, 2001).

Além disso, uma grande dificuldade para os profissionais que atuam nas empresas é a falta de pesquisas orientadas para avaliar o desempenho e otimização da logística florestal. A realização de pesquisas que visem à organização, racionalização das operações do transporte florestal rodoviário, identificação dos fatores operacionais e interferência no custo, poderão representar uma grande economia de recursos para as empresas do setor, aumentar a eficiência operacional, a produtividade dos veículos, bem como dar a confiabilidade necessária para a tomada de decisão adequada.

Sendo assim, objetivou-se com esta pesquisa realizar uma análise dos fatores técnicos e de custos do transporte de madeira com diferentes composições veiculares de carga, na região do Vale do Rio Doce, Estado de Minas Gerais.

\section{MATERIAL E MÉTODOS}

\subsection{Região de estudo}

O estudo foi realizado em três regiões do Vale do Rio Doce, localizadas no Leste do Estado de Minas Gerais. A região de Guanhães, com distância média de projetos de $135 \mathrm{~km}$ da fábrica (localizado no município de Belo Oriente), na qual o transporte de madeira era realizado por tritrem e rodotrem.Região de Nova Era, com distância média de projetos de $74 \mathrm{~km}$ da fábrica, utilizando bitrem como veículo de transporte e a região do Rio Doce, com distância média de projetos de 73 km da fábrica, com utilização de bitrem e tritrem para baldeio da madeira.

\subsection{Caracterizações dos veículos utilizados por região}

Os veículos utilizados para avaliação do estudo foram o bitrem articulado com comprimento de 19,8 m e PBTC de 57 toneladas, triitrem com comprimento de 30,00 m e PBTC de 74t., e rodotrem com 30 m de comprimento e PBTC de 74t.

As operações de carregamento foram subdivididas em carregamento de madeira de 6,0 m e madeira de 2,2 m, sendo que a madeira de 2,2 m em sua totalidade é transportada pelo veículo Rodotrem, que comporta 8 feixes totalizando uma carga líquida média de $65 \mathrm{~m}^{3}$. A madeira de 6,0 m é transportada preferencialmente pelo tritrem, que comporta 3 feixes e totaliza uma carga líquida média de 58,5 m³. Porém, os dois veículos possuem a mesma capacidade de carga em toneladas, o que ocasiona o transporte igual em peso.

\subsection{Análise dos fatores técnicos}

Inicialmente realizou-se um estudo de tempo e movimentos do transporte nas diferentes regiões estudadas pelo método de tempos contínuos. Nessas 
condições, o ciclo operacional do transporte foi subdividido nos seguintes componentes parciais:

- Viagem vazio:corresponde ao tempo consumido com o deslocamento do veículo de transporte da fábrica até o ponto de carregamento

- Carregamento:corresponde ao tempo consumido no carregamento dos diferentes tipos de madeira (madeira com casca e sem casca) nos veículos de transporte.

- Viagem carregado:corresponde ao tempo consumido com o deslocamento do veículo de transporte do ponto de carregamento no campo até a balança da fábrica.

- Descarregamento:corresponde ao tempo consumido no descarregamento dos diferentes tipos de madeira nos veículos de transporte, compreendendo desde a entrada do veículo na área fabril até a sua saída, e

- Pausas/Interrupções: é qualquer outro tempo não mencionado acima e queinterfere na realização do transporte da madeira.

Para a realização do estudo de tempos e movimentos, foi utilizado um cronômetro digital e formuláriosespecíficos para o registro dos dados. O acompanhamento das operações foi realizado in loco, sem qualquer interferência.

Para a avaliação do estudo de desempenho operacional, os tempos gastos com cada etapa operacional foram divididos a saber:

- Tempo Produtivo (TP): é o tempo quando o veículo está efetivamente desempenhando a sua função produtiva;

- Tempo Auxiliar (TA): é o tempo despendido com funções auxiliares (como por exemplo recebimento de notas fiscais), obrigatoriamente exigidas pela operação.

- Tempo Acessório (TAc): é o tempo despendido com funções obrigatórias, porém não diretamente relacionadas com a operação, como paradas pessoais;

- Tempo Improdutivo (TI): é o tempo despendido em que o veículo está disponível para a operação, porém não é utilizado em função de aspectos gerenciais do sistema, independentes do veículo, máquina ou implemento florestal utilizados no carregamento ou descarregamento; e

- Tempo em Manutenção (TM): é o tempo despendido com a manutenção preventiva ou corretiva do veículo.

\subsubsection{Amostragem}

Para o estudo dos tempos e movimentos, os resultados de cada ciclo foram avaliados de forma a obter-se a amostragem dentro do proposto pelo estudo, respondendo a $90 \%$ da operação real e normal. A amostragem mínima foi definida através de um estudo piloto, por meio da seguinte expressão proposta por Conaw (1977), citado por Minette (1996):

$$
n \geq \frac{t^{2} * s^{2}}{e^{2}}
$$

Em que: $\mathrm{n}$ = número de amostras ou pessoas necessárias; $\mathrm{t}$ = valor tabelado a $10 \%$ de probabilidade (distribuição t de Student); s = desvio-padrão da amostra; e $=$ erro admissível a $10 \%$ em torno da média.

\subsubsection{Disponibilidade mecânica}

A disponibilidade mecânica foi obtida a partir da relação entre o tempo total de trabalho destinado para as atividades, quando o veículo encontrava-se apto para o desempenho de suas funções e o período em que o trabalho era interrompido para manutenção. O cálculo foi obtido pela seguinte expressão:

$$
D M=\frac{H T-H M}{H T} * 100
$$

Em que: $\mathrm{DM}=$ disponibilidade mecânica; $H T=$ horas totais de trabalho e $H M=$ horas em manutenção.

As horas em manutenção corretiva foram compostas pelos tempos que o veículo ficou inoperante devido a problemas mecânicos, como: pneu furado, problemas no freio, problemas no motor, etc. As fases de manutenção preventiva e corretiva não foram computadas nesse cálculo, pois teoricamente quando um veículo entra nesse processo, ele é automaticamente substituído no sistema por um outro, de modo a manter sempre o número constante de veículos.

\subsubsection{Eficiência operacional}

A eficiência operacional é compreendida pelo dimensionamento da capacidade horária total de produção do veículo e mão-de-obra pelo tempo em que o veículo esteve efetivamente realizando o transporte. Muitas vezes, a eficiência operacional está relacionda com as características de trabalho do operador, como o tempo para as refeições, pausas, higiene pessoal, condições de operação, estrada, tipo de povoamento. 
A eficiência operacional foi obtida pela seguinte expressão:

$$
E 0=\frac{T e f}{T e f+H i} * 100
$$

Em que: $\mathrm{EO}$ = eficiência operacional em \%;Tef = tempo de trabalho efetivo em horas; e $\mathrm{Hi}=$ horas de interrupções operacionais.

Após avaliação detalhada, o ciclo de transporte foi dividido em operações comuns a todas as regionais e tipo de veículos, e em fases dentro das operações, devido às características locais, de veículos e obrigatoriedades a serem cumpridas.

\subsection{Análise de custos do transporte}

A análise de custos do transporte foi realizada pelo método North American (1956), proposto por Freitas et al. (2004) e adaptado para a realidade do estudo, conforme descrito abaixo.

\subsubsection{Custos fixos}

\subsubsection{Depreciação}

$$
A=\frac{B+C+D}{E * F}
$$

Em que: A) custo da depreciação por km; B) custo do veículo com pneu; C) custo da carreta sem pneu; D) valor residual - 50\%; E) tempo de depreciação 36 meses; F) quilometragem média mensal.

\subsubsection{Salário Motorista + Encargos Sociais (S.M.)}

$$
S . M=\frac{A *(B+C+D)}{E}
$$

Em que: A) número de motoristas; B) salário fixo mensal; C) encargos sociais mensais - 77\%; D) diária -R\$; E) quilometragem média mensal.

\subsubsection{Licenciamento / Seguro Obrigatório(L.S.)}

$$
L . S=\frac{A}{B * C}
$$

Em que: A) seguro; B) número de meses; C) quilometragem média mensal.

\subsubsection{Remuneração de Capital (R.C.)}

$$
\text { R.C. }=\frac{0,1 * A}{B}
$$

Revista Árvore, Viçosa-MG, v.37, n.5, p.897-904, 2013
Em que: A) investimento - (veículo + carreta); B) quilometragem média mensal.

\subsubsection{Seguro do casco (S.C.)}

$$
\text { S.C. }=\frac{A \times B}{C * D}
$$

Em que: A) taxa sobre investimento - 7\%; B) valor do investimento (veículo + carreta); C) número de meses -12 ; D) quilometragem média mensal.

\subsubsection{Equipamentos de segurança (E. S.)}

$$
E . S .=\frac{A+B+C}{D}
$$

Em que: A) camisas, botas, calças, luvas (03 unidades por semestre); B) capacete, capa de chuva (03 unidades por semestre por motorista); C) despesa média mensal; D) quilometragem média mensal.

\subsubsection{Custos variáveis}

\subsubsection{Peças e materiais de reposição (P.M.)}

$$
\text { P.M. }=\frac{A * B}{C}
$$

Em que: A) total do investimento (veículo + carreta); B) índice: 1,3\% a.m.; C) quilometragem média mensal.

\subsubsection{Pneus / câmaras / protetores / recapagem (P)}

$$
P=\frac{(A+B+C+D) * F}{E}
$$

Em que: A) pneus novo; B) câmara de AR; C) protetor; D) recapagem; E) quilometragem por pneu; F) número de pneus.

\subsubsection{Combustível (C.)}

$$
\text { C. }=\frac{A}{B}
$$

Em que: A) preço por litro; B) consumo médio de $1,2 \mathrm{~km} / \mathrm{litro}$

Nota: O preço do combustível por litro era o mesmo paras as regionais avaliadas.

\subsubsection{4. Óleo de câmbio (O.C.)}

$$
\text { O.C. }=\frac{A * B}{C}
$$


Em que: A) quantidade de litros; B) preço por litro; C) quilometragem para troca $-35.000 \mathrm{~km}$.

\subsubsection{5. Óleo de diferencial (O.D.)}

$$
\text { O.D. }=\frac{A * B}{C}
$$

Em que: A) quantidade de litros - 18 litros; B) preço por litro; C) quilometragem para troca $-50.000 \mathrm{~km}$.

\subsubsection{6. Óleo de motor (O.M.)}

$$
O \cdot M .=\frac{(A+D) * B}{C}
$$

Em que: A) quantidade de litros - 28 litros; B) preço por litro; C) quilometragem para troca -10.000 $\mathrm{km}$; D) consumo a cada $1.500 \mathrm{~km}$.

\subsubsection{Lavagem e lubrificação (L)}

$$
L=\frac{A}{B}
$$

Em que: A) preço de cada lavagem e lubrificação; B) pedido $-1.932 \mathrm{~km}$.

O custo total do transporte florestal foi obtido pelo somatório dos custos fixos, variáveis e administrativo (6\% sobre os custos fixos e variáveis). Após obtido os custos de transporte por km, dividiu-se pelo volume de carga de madeira transportada, para encontrar o custo do metro cúbico de madeira transportada por $\mathrm{km}$.

\section{RESULTADOS}

Os resultados referentes às análises técnica e de custo do transporte realizado com os diferentes tipos de veículos são apresentados em conjunto dentro de cada região estudada.

No período diurno foram coletadas 15 amostras, quando eram necessárias nove amostras; no período noturno foram coletadas 10 amostras, sendo este, o mínimo necessário.

Na Tabela 1, estão os resultados consumidos durante o transporte florestal nas três regionais estudadas.

\subsection{Região de Guanhães}

Para melhor compreensão e análise dos resultados, os tempos de viagem do veículo de transporte na região de Guanhães foram subdivididos, podendo-se observar o tempo consumido para cada fase operacional (Tabela 2).

\subsection{Região de Nova Era}

Na região de Nova Era, durante o período diurno foram coletadas 17 amostras de ciclo operacional, sendo necessárias 13; no período noturno foram coletadas 12 amostras, sendo necessárias somente 2 .

O transporte de madeira na região de Nova Era é considerado na empresa como “diferenciado” em decorrência do carregamento de madeira em dois pontos diferentes no campo, normalmente o primeiro carregamento é realizado na região de maior dificuldade de manobras do veículo na área, e posteriormente a carga é completada numa área de melhor acesso, ou na saída do projeto (Tabela 3).

\subsection{Região do Rio Doce}

Durante o período do estudo, as viagens com o bitrem só foram realizadas no período diurno, portanto, para a comparação foram filtrados os valores do tritrem para terem as mesmas bases de análise.Foram coletadas 23 amostras de ciclo operacional para o bitrem, sendo necessárias 12 amostras; enquanto para o tritrem foram coletadas 23 amostras, sendo necessárias 16 .

Para melhor compreensão e análise dos resultados, os tempos foram subdivididos podendo-se observar o tempo consumido para cada fase operacional, como apresentado na Tabela 4.

A Tabela 4refere-se à estratificação dos tempos de viagem para os veículos bitrem e tritrem na região Rio Doce.

\subsection{Comparação entre as regionais e o tipo de transporte}

Com base nos resultados obtidos para cada região e tipo de transporte, é possível estabelecer uma comparação entre os indicadores de rendimento do transporte e oscustos médios da madeira posta em fábrica para cada região (Tabela 5).

\section{DISCUSSÕES}

\subsection{Região de Guanhães}

Como pode ser visto na Tabela 2, a eficiência do sistema de transporte na região estudada foi de $90,41 \%$, sendo considerado como o somatório do tempo

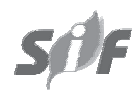

Revista Árvore, Viçosa-MG, v.37, n.5, p.897-904, 2013 
Tabela 1 - Distribuição dos tempos consumidos na etapa do transporte florestalna região de Guanhães, Nova Era e Rio Doce. Table 1 - Distribution of times spentin the stage of forest transportation in theregion of Guanhães, Nova Era and Rio Doce.

\begin{tabular}{|c|c|c|c|c|}
\hline \multirow{3}{*}{ Classificação dos tempos } & \multicolumn{4}{|c|}{ Porcentagem (\%) } \\
\hline & \multirow{2}{*}{ Guanhães } & \multirow{2}{*}{ Nova Era } & \multicolumn{2}{|c|}{ Rio Doce } \\
\hline & & & bitrem & tritrem \\
\hline Tempo Produtivo & 75,77 & 78,71 & 71,48 & 68,25 \\
\hline Tempo Auxiliar & 6,39 & 9,66 & 10,81 & 13,91 \\
\hline Tempo Acessório & 6,96 & 0,13 & 7,29 & 1,65 \\
\hline Tempo Improdutivo & 6,60 & 9,26 & 4,38 & 6,86 \\
\hline Tempo em manutenção & 2,15 & 2,24 & 5,24 & 5,91 \\
\hline Outros & 2,14 & 0,00 & 0,80 & 3,42 \\
\hline
\end{tabular}

Tabela 2 - Estratificação dos tempos de viagem na região de Guanhães.

Table 2 - Stratification of travel time in the region of Guanhães.

\begin{tabular}{lc}
\hline Atividade & Porcentagem (\%) \\
\hline Viagem vazio* & 31,75 \\
Viagem carregado* & 44,02 \\
Carregamento (Madeira 6,0 m)* & 1,92 \\
Carregamento (Madeira 2,2 m)* & 2,18 \\
Reaperto de carga** & 2,29 \\
Parada Obrigatória*** & 1,97 \\
Balança*** & 2,34 \\
Deslocamento dentro do carregamento** & 0,20 \\
Parada Apoio*** & 2,44 \\
Espera carregamento**** & 3,60 \\
Parada**** & 3,00 \\
Problemas Mecânicos***** & 0,73 \\
Atolado**** & 1,42 \\
Pista Interditada**** & 0,84 \\
Almoço*** & 1,14 \\
Troca de turno*** & 0,16 \\
Total & 100 \\
\hline
\end{tabular}

Nota: *Eventos caracterizados como tempo produtivo; ** eventos caracterizados como tempos auxiliares; *** eventos caracterizados como tempos acessórios; ****eventos caracterizados como tempos improdutivos; *****eventos caracterizados como tempos de manutenção.

produtivo, tempo acessório e tempo auxiliar (retirando os tempos improdutivos e de manutenção como paradas, problemas mecânicos, espera para carregamento, atolamento e pista interditada). Em relação ao tempo de carregamento, verifica-se que esta operação consumiu 4,1\% do total do tempo do ciclo operacional. Foi possível ainda verificar que, o tempo de espera do veículo de transporte para o carregamento consumiu 3,6\% do tempo total, representando em média 3 minutos, sendo considerado elevado e podendo afetar a eficiência do transporte.

Revista Árvore, Viçosa-MG, v.37, n.5, p.897-904, 2013
Dentro dos fatores acessórios, somente o tempo consumido com paradas nas balanças rodoviárias são difíceis de reduzir, os outros três fatores podem e devem ser melhorados, como as paradas obrigatórias e paradas nos pontos de apoio, seguindo corretamente a programação disponibilizada pela empresa contratante: essas paradas tendem a diminuir e em alguns trechos até deixar de existir. Essas paradas são necessárias para que não ocorra o chamado comboio na estrada, que é quando por denominação da empresa, três ou mais veículos trafegam próximos (distância inferior a $300 \mathrm{~m}$ entre veículos), caso isso seja constatado, a operadora logística é notificada correndo o risco de sofrer penalidades constantes em contrato.

Em relação aos tempos improdutivos ou perdidos constatou-se que estes totalizam 6,6\% do tempo total observado, somente nesse ponto. Dentro dos tempos perdidos, $3 \%$ são pausas que ocorrem sem justificativa operacional ou fisiológica por parte do motorista.

\subsection{Região de Nova Era}

A eficiência do sistema de transporte da região totaliza 88,5\% (Tabela 3), que é o somatório do tempo produtivo, tempo acessório e tempo auxiliar.

Um ponto muito importante e que exige atuação rápida está relacionado aos tempos improdutivos, que somam 9,26\% do tempo total da viagem. Somente o tempo de espera por carregamento foi 7,1\% do tempo total, isso demonstra um gargalo na operação e é causado principalmente pelo não cumprimento por parte da operadora logística da programação de saída de veículo disponibilizada pela empresa. Outro ponto dentro dos tempos improdutivos são as paradas sem justificativas operacionais ou fisiológicas, que somam 2,2\% do tempo 
Tabela 3 - Estratificação dos tempos de viagem na região de Nova Era.

Table 3 - Stratification of travel time in the region of Nova Era.

\begin{tabular}{lc}
\hline Atividade & Porcentagem (\%) \\
\hline Viagem Vazio* $^{*}$ & 30,8 \\
Viagem Carregado* & 47,9 \\
Carregamento $1^{\circ}$ Ponto* $^{*}$ & 5,7 \\
Carregamento $^{\circ}$ Ponto* $^{*}$ & 0,8 \\
Reaperto de carga** & 3,1 \\
Deslocamento entre carregamento** & 0,1 \\
Espera carregamento**** & 7,1 \\
Parada**** & 2,2 \\
Pneu furado***** & 2,2 \\
Total & 100 \\
\hline
\end{tabular}

Nota: *Eventos caracterizados como tempo produtivo; ** eventos caracterizados como tempos auxiliares; *** eventos caracterizados como tempos acessórios; ****eventos caracterizados como tempos improdutivos; *****eventos caracterizados como tempos de manutenção.

Tabela 4 - Estratificação dos tempos de viagem para bitrem e tritrem na região do Rio Doce.

Table 4 - Stratification of travel time for bitremand tritrem trucks in region of Rio Doce.

\begin{tabular}{lcc}
\hline Atividade & $\begin{array}{c}\text { bitrem } \\
(\%)\end{array}$ & $\begin{array}{c}\text { tritrem } \\
(\%)\end{array}$ \\
\hline Viagem Vazio* & 32,81 & 30,46 \\
Viagem Carregado* & 38,67 & 37,80 \\
Carregamento* & 7,03 & 5,97 \\
Reaperto de Carga** & 3,79 & 7,94 \\
Deslocamentoentre carregamento** & 1,60 & 1,23 \\
Parada Obrigatória*** & 5,69 & 0,33 \\
Espera carregamento**** & 4,25 & 6,67 \\
Parada**** & 0,13 & 0,18 \\
Parada Pátio**** & 5,2 & 4,50 \\
Problemas Mecânicos***** & 0,00 & 0,59 \\
Atolado**** & 0,00 & 0,83 \\
Pista Interditada**** & 0,59 & 1,22 \\
Almoço*** & 0,00 & 1,90 \\
Troca de turno*** & 0,15 & 0,30 \\
Total & 100 & 100 \\
\hline
\end{tabular}

Nota: *Eventos caracterizados como tempo produtivo; ** eventos caracterizados como tempos auxiliares; *** eventos caracterizados como tempos acessórios; ****eventos caracterizados como tempos improdutivos; *****eventos caracterizados como tempos de manutenção. total, coincidentemente o mesmo percentual do tempo parado por problemas mecânicos.

\subsection{Região do Rio Doce}

Durante todo o período avaliado, só houve transporte de madeira de 6,0 m nesta Região. Como pode ser observado na Tabela 4 o tempo de carregamento consumiu mais tempo nas viagens do bitrem $(7,03 \%)$, porém, o tempo de aperto de carga durante a viagem foi menor (3,79\%) quando comparado ao tritrem (7,94\%). O tempo perdido por espera para carregamento na viagem do bitrem (4,25\%), também foi menor quando comparado ao tritrem (6,67\%). Isto pode ser explicado pelo fato do bitrem possuir uma capacidade de carga de $57 \mathrm{~m}^{3}$ (33\% menor em relação ao tritrem), assim o tempo necessário para o carregamento é menor.

O bitrem apresentou eficiência operacional de 89,58\% e o tritrem de $83,81 \%$ que éo somatório do tempo produtivo, tempo acessório e tempo auxiliar; a eficiência mínima exigida pela empresa é de 85\%.Assim, o tritrem apresentou eficiência operacional abaixo do mínimo exigido.

A disponibilidade mecânica do bitrem também foi maior que a do tritrem, 94,76\% e 94,09\%, respectivamente. Porém, na sua totalidade o tempo de manutenção do bitrem foi decorrência de ajustes necessários pela adaptabilidade ao novo sistema.

A maior eficiência aparente do sistema com bitrem foi observada em função da utilização dos mesmos cavalos mecânicos dos tritrem. Como o bitrem possui capacidade de carga menor, houve favorecimento para o aumento no desempenho dos veículos, logo de todo o sistema.

\subsection{Comparação entre as regionais e $o$ tipo de transporte}

Como pode ser observado na Tabela 5, a utilização do tritrem na região de Guanhães e o bitrem no Rio Doce apresentaram os melhores indicadores de eficiência

Tabela 5 - Comparativo entre regiões e veículos de transporte.

Table 5 - Comparison betweenregions andtransportvehicles.

\begin{tabular}{lcccc}
\hline REGIÃO & EF & DM & Custo $\left(\mathrm{R} \$ / \mathrm{m}^{3} \cdot \mathrm{km}^{-1}\right)$ & $\mathrm{CMF}\left(\mathrm{R} \$ \cdot \mathrm{m}^{-3}\right)$ \\
\hline Guanhães - tritrem & $89,12 \%$ & $97,85 \%$ & 0,201 & 27,14 \\
Nova Era - bitrem & $88,50 \%$ & $97,80 \%$ & 0,341 & 25,23 \\
Rio Doce - tritrem & $83,81 \%$ & $94,09 \%$ & 0,208 & 15,18 \\
Rio Doce - bitrem & $89,58 \%$ & $94,76 \%$ & 0,249 & 18,18 \\
\hline
\end{tabular}


operacional, porém ao observar a disponibilidade mecânica, verificou que o tritrem para a região de Guanhães apresentou melhores resultados, visto que, apresentou o menor percentual com tempos mecânicos (2,15\%, Tabela 5).

Em relação ao custo de transporte da madeira, o maior custo foi obtido para a região de Guanhães, visto essa região ser a mais afastada da unidade fabril da Empresa (distância média de 135 km).

Porém, ao analisar somente o custo do metro cúbico de madeira por $\mathrm{km}$, verificou-se que o maior custo, foi obtido utilizando o veículo bitrem, pelo fato deste transportar menor volume por ciclo de transporte (54 toneladas, aproximadamente $33 \%$ a menos que o transporte via tritrem). Além disso, o valor obtido na região de Nova Era foi maior $\left(0,341 \mathrm{R} \$ / \mathrm{m}^{3} \cdot \mathrm{km}^{-1}\right)$ devido esta região obter o maior percentual com tempos improdutivos $(9,26 \%)$.

\section{CONCLUSÕES}

A região de Nova Era apresentou o melhor percentual de tempo produtivo, porém obteve o pior resultado com tempos improdutivos em relação a região de Guanhães devido ao alto percentual de tempo gasto do veículo com espera para carregamento;

Os maiores valores de indicadores de rendimento (Disponibilidade Mecânica e Eficiência Operacional) foram obtidos na região de Guanhães;

A utilização do tritrem como veículo para transporte de madeira apresentou os menores custos de $\mathrm{m}^{3}$ transportado por km;

O maior custo de madeira posto em fábrica, foi obtido pela região de Guanhães, devido apresentar a maior distância em relação à unidade fabril. Ressaltando a importância de que, quanto maior for a distância de transporte de madeira, deve se utilizar veículos com capacidade de carga maiores, afim de reduzir o custo final do $\mathrm{m}^{3}$ de madeira.

\section{REFERÊNCIAS}

BERGER, R. et al.Minimização de custos de transporte florestal com a utilização da programação linear. Revista Floresta, v.33, n.1, p.53-62, 2003.

BURLA, E. R. Mecanização de atividades silviculturais em relevo ondulado. Belo Oriente: 2001.144p.

FREITAS, L.C. et al. Estudo comparativo envolvendo três métodos de cálculo de custo operacional do caminhão bitrem. Revista Árvore, v.28, n.6, p.855-863, 2004.

MACHADO, C.C.; LOPES, E.S.; BIRRO, M.H. Elementos básicos do Transporte Florestal Rodoviário. 2.ed. Viçosa, MG: Universidade Federal de Viçosa, 2009.167p.

MinetTE, L. J. Análise de fatores operacionais e ergonômicos na operação de corte florestal com motosserra. 1996. 211f. Tese (Doutorado em Ciência Florestal) - Universidade Federal de Viçosa, Viçosa, MG, 1996.

RODRIGUES, P. Introdução aos sistemas de transporte no Brasil e à logística internacional. 4.ed.São Paulo: Aduaneiras, 2007. 